\title{
LAS NUEVAS TECNOLOGÍAS COMO INSTRUMENTOS DIDÁCTICOS EN NUESTRO QUEHACER DOCENTE
}

MSc. Marcos Hernández Zamora

Correo: marcos.hernandez@norte.uni.edu.ni

Docente UNI -Norte
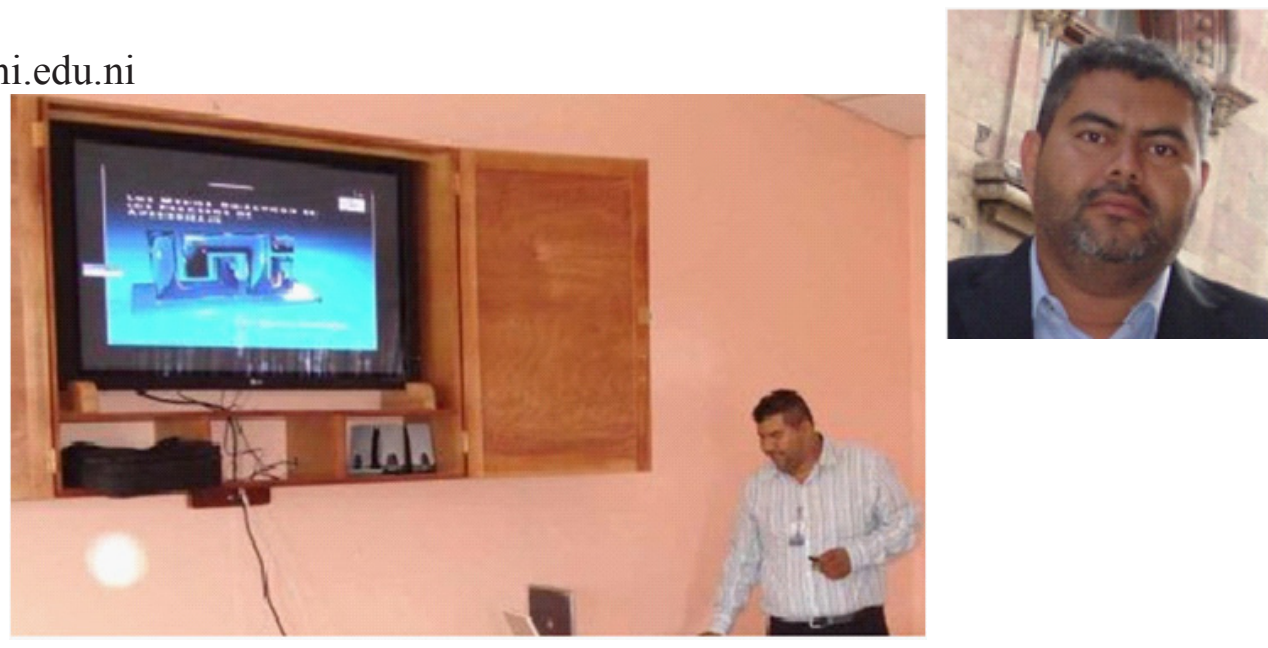

Las computadoras, tal y como la conocemos hoy, tienen su aparición a inicios de la década de los 80 , cuando se estaba apostando por un uso personal, fuera de las grandes corporaciones y empresas que podían costear un súper equipo. A muchos les pareció "ideas de locos" que una computadora pudiera ser de utilidad en un hogar, mucho menos se imaginarían que podría ser utilizada para la enseñanza en las escuelas e instituciones de educación superior como un instrumento más a disposición de los profesores.

Por otro lado: "Si hasta hace relativamente poco tiempo la influencia de las denominadas nuevas tecnologías de la información y comunicación se centraban fundamentalmente en los sectores militares, bancarios y de transferencia de comunicación de masas, en poco tiempo su impacto está alcanzando a todos los sectores de la sociedad, desde la enseñanza a la medicina, y desde el mundo del arte a la investigación" (Julio Cabero, 2003).

Realmente como profesores debemos aprovechar todo lo disponible en nuestro entorno para apoyarnos en nuestras labores relacionadas con el proceso enseñanza aprendizaje. Por esto, se debe hacer la aclaración que la mayoría de los instrumentos didácticos presentes a nuestro alrededor, no fueron concebidos para la función educativa y algunos de ellos, son parte de nuestro entorno desde hace mucho tiempo: lugares históricos y monumentos naturales como el Cañón de Somoto y la montaña del Tisey. Sin embargo, otros, están haciendo su aparición en la historia reciente y son las denominadas
Tecnologías de la Información y la Comunicación (TICs). Con esto queda aclarado que no existe un instrumento didáctico elaborado especialmente para una asignatura específica o lo que es más, para un tema específico.

Apegándonos al concepto de didáctica: "Es una ciencia y un arte que contribuye en el proceso enseñanza aprendizaje, aportando estrategias educativas que permiten facilitar el aprendizaje.” (psicopedagogia, 2005), como educadores debemos procurar incorporar los instrumentos de las TICs, no sólo para cumplir con los requerimientos que como docentes estamos obligados, sino para que hagamos de nuestro quehacer más que una ciencia un arte.

Debemos recordar que la sociedad ha adoptado las ventajas de las TICs, para su mejor aprovechamiento de la calidad de vida. La región Segoviana no ha sido la excepción y muchos tenemos tecnología de punta en nuestros hogares, las sedes de universidades de la región en su equipamiento tienen TICs (computadoras con accesorios de multimedia, datas, acceso a internet, teléfonos, equipos de audio y video, etc.).

Lo antes expuesto nos lleva a lo siguiente: ¿Qué podría impedir a los profesores de las universidades de la región utilizar estos medios y convertirlos en instrumentos didácticos? Podemos plantear algunas razones, que analizadas desde una perspectiva cultural nos permitirán comprender las causas. 
Primero, tomemos en cuenta si los profesores tienen una formación tecnológica adecuada para el uso de estos instrumentos. De no ser así, esto limitaría el uso de instrumentos tecnológicos como instrumentos didácticos, cuyo uso mejoraría de manera significativa el proceso enseñanza aprendizaje.

En segundo lugar, son necesarias las capacitaciones para desarrollar competencias en el uso de estos instrumentos convertidos en didácticos. Estas capacitaciones necesitan tiempo y disponibilidad, lo que nos lleva a la siguiente causa.

Tercero, determinar cuanta voluntad existe de parte del profesorado al verse implicado en la mejora continua de su práctica profesional, la cual se deriva de la transformación constante de la sociedad en el aspecto tecnológico. La evolución que se vive fuera de los centros de estudio con respecto a la tecnología no debe ser ajena a nuestra vida profesional como docentes.

Un último aspecto se correspondería con la presencia de los medios en los centros de estudio y los aspectos organizativos para la integración de los medios (Compostela, 2002). Así, planteo las siguientes interrogantes, ¿Qué instrumentos tecnológicos existen en el centro y cuáles de estos están disponibles para que los profesores los utilicen en sus clases? Y ¿Qué grado de eficiencia de la organización de horarios existe para el mejor aprovechamiento de las sesiones de clases con estos instrumentos?

La comunidad educativa debe sensibilizarse respecto a estos nuevos retos y proporcionar alternativas en cuanto a modalidades de aprendizaje (Cabero, 2001). Los profesores al tomar conciencia de los cambios que se producen en la sociedad con las nuevas tecnologías, debemos ser pertinentes en su uso. De tal forma que si un profesor utiliza un blog, el cual es un sitio web donde se informa, comparte, y debate periódicamente sobre un tema (Aulablog, 2005); para interactuar con sus estudiantes y discutir el contenido y forma de la clase, está utilizando una tecnología como instrumento didáctico, agregando capacidades de búsqueda e interacción al estudiante, quien en un futuro, estará en una posición ventajosa en su desempeño profesional.

La universidad Nacional de Ingeniería Sede Regional Estelí (UNI - Norte), consciente de la importancia del uso de la nueva tecnología como instrumento didáctico, comenzó a impulsar desde inicio de sus funciones (2005), la inclusión de datas, laboratorios de cómputo, software especializados y páginas web, para desarrollar las distintas asignaturas de los Planes de Estudio de las cuatro carreras de ingeniería de la UNI - Norte.

A inicios del año 2008 en el primer semestre, los profesores de la UNI - Norte contaban con tres Datas móviles con PC Portátiles para presentaciones de sus clases en las aulas, dos aulas equipadas con televisores de plasma de 42 pulgadas, una sala de medios equipada con un $\mathrm{PC}$, un televisor de plasma de 50 pulgadas y un equipo de audio interconectados entre sí para las clases que necesitan multimedia, un escáner y dos laboratorios de cómputos. Según el registro de la oficina de informática de la Sede, los profesores hicieron uso de los distintos medios según gráfica mostrada a continuación.

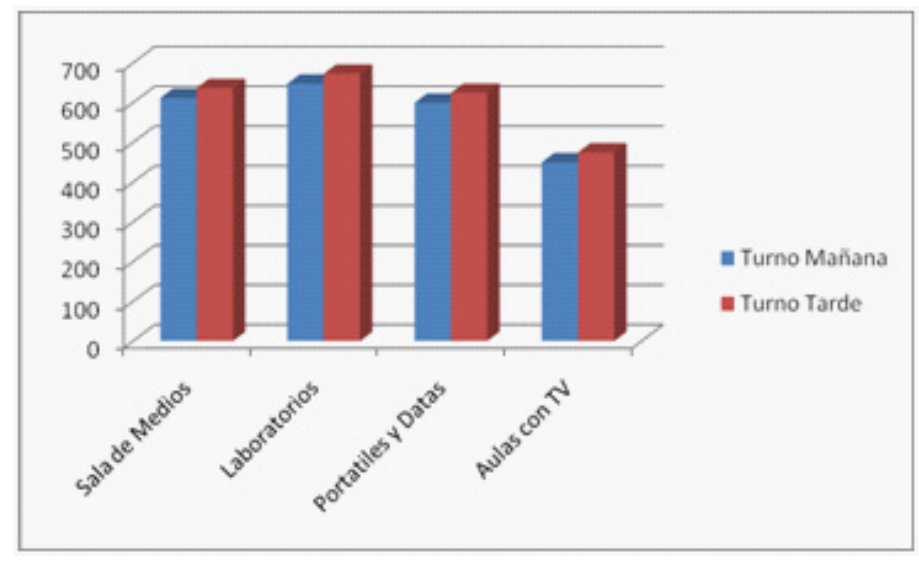

Durante el segundo semestre la Dirección de la Sede impulsa la iniciativa del uso de blogs para las distintas asignaturas. Se inicia con una capacitación a los profesores: 48 profesores. Después de la capacitación 39 profesores, $81 \%$, hicieron uso de blogs para interactuar con sus estudiantes.

Las ventajas para los docentes que utilizaron esta tecnología fueron, esencialmente, proporcionar en línea todos los documentos elaborados por ellos, crear foros de discusión sobre temas específicos. Los estudiantes pudieron acceder al material a cualquier hora, pero lo más importante radica en la disposición de las presentaciones en el sitio. Pueden consultar al profesor sobre diversidad de tópicos que no pudieron o no pueden ser abordados por los docentes en el aula, debido al factor tiempo. 
Idealmente deberíamos contar con el cien por ciento de profesores utilizando blogs, pero estamos ante un reto, uno que nos lleva a evaluar nuestra condición de educadores profesionales. Como tales, debemos estar abiertos al cambio, máxime si este cambio nos lleva a la adquisición de competencias que hagan nuestro trabajo pertinente a las necesidades de nuestra sociedad actual.

\section{BIBLIOGRAFÍA:}

Aulablog. (11 de Abril de 2005). AulaBlog.com. Recuperado el 28 de Enero de 2009, de http://www.aulablog. $\mathrm{com} /$

Julio Cabero, C. C. (12 de Enero de 2003). Revista Pixel Bit. Recuperado el 16 de Enero de 2009, de Revista Pixel Bit. Número 20. Enero del 2003: http:// www.sav.us.es/pixelbit/pixelbit/articulos/n20/n20art/ art2008.htm

Psicopedagogia. (11 de Marzo de 2005). psicopedagogia.com. Recuperado el 28 de Enero de 2009, de http:// www.psicopedagogia.com/: http://www.psicopedagogia.com/definicion/didactica

Tecnología educativa / coord. por Julio Cabero Almenara, 2007, ISBN 84-481-5613-7

Innovación educativa, Santiago de Compostela, 2002, ISBN 00-820-0330-038

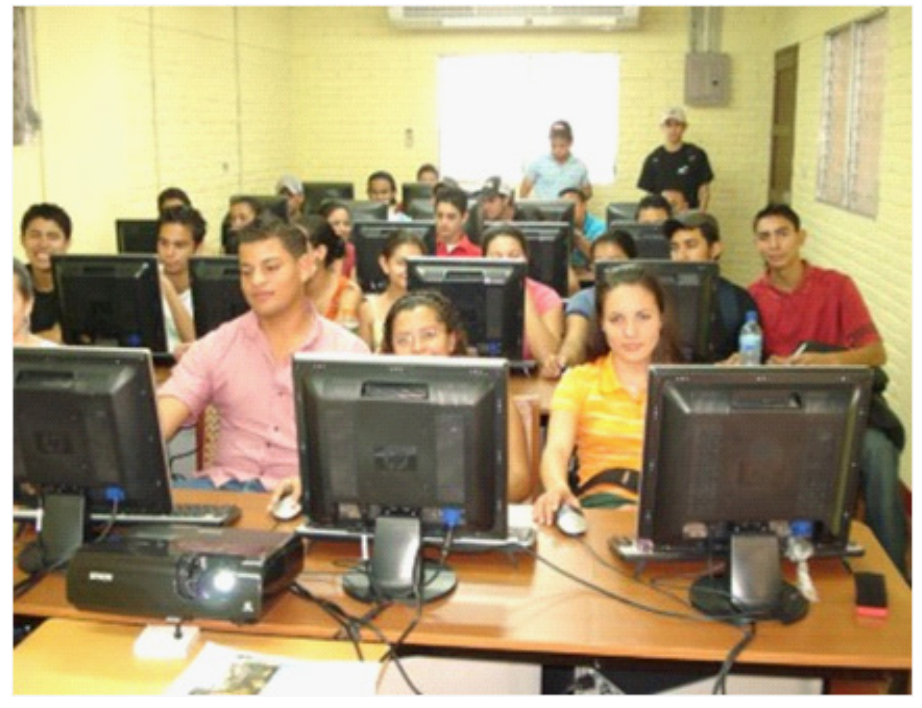

Laboratorio B de Cómputo UNI - Norte

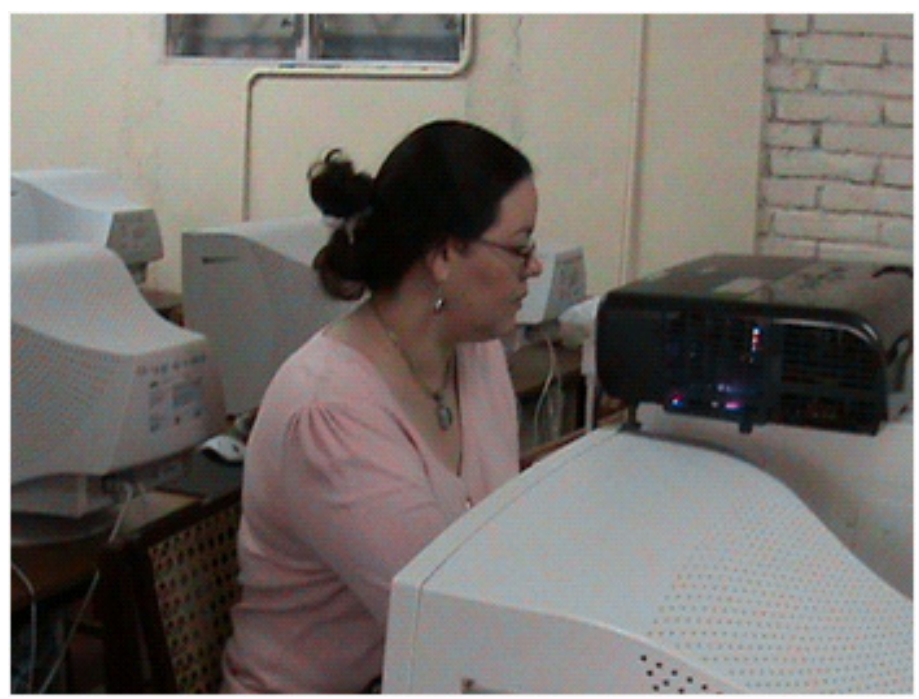

Docente UNI Norte utilizando computadora Laboratorio A

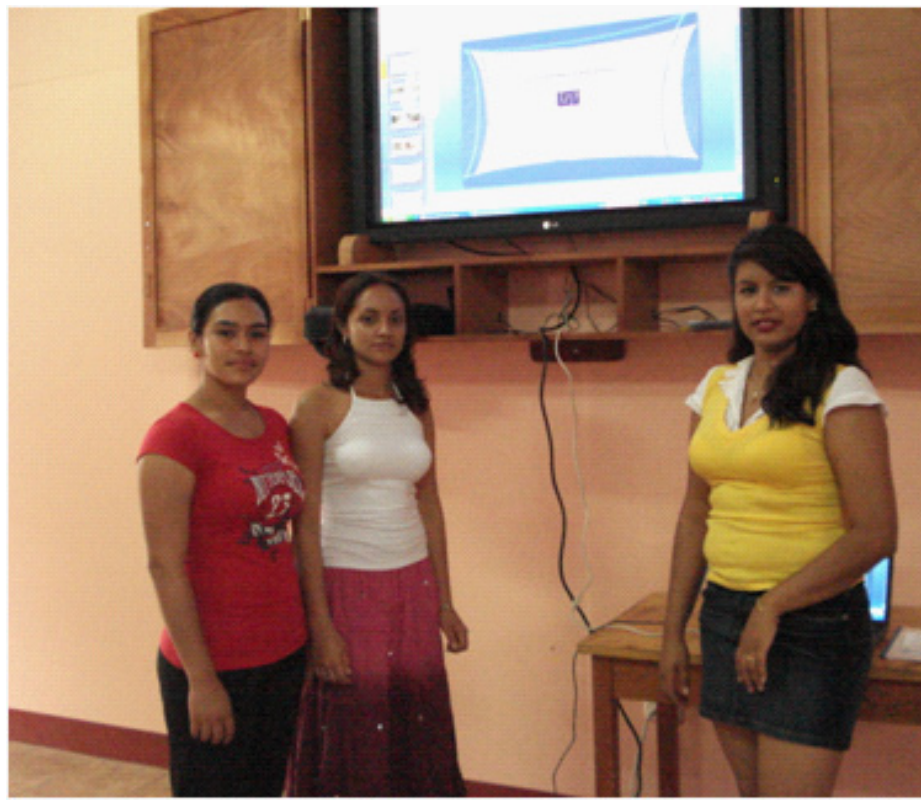

Estudiantes de UNI Norte realizando exposiciones en Aula con medios AudioVisuales. 